\title{
Impaired SUMOylation of nuclear receptor LRH-1 promotes nonalcoholic fatty liver disease
}

\author{
Sokrates Stein, ${ }^{1,2}$ Vera Lemos, ${ }^{1,3}$ Pan Xu, ${ }^{1}$ Hadrien Demagny, ${ }^{1}$ Xu Wang, ${ }^{4}$ Dongryeol Ryu, ${ }^{4}$ Veronica Jimenez, ${ }^{5}$ Fatima Bosch, ${ }^{5}$ \\ Thomas F. Lüscher, ${ }^{2}$ Maaike H. Oosterveer, ${ }^{6}$ and Kristina Schoonjans ${ }^{1}$ \\ 'Laboratory of Metabolic Signaling, Institute of Bioengineering, School of Life Sciences, École Polytechnique Fédérale de Lausanne, Lausanne, Switzerland. 2Center for Molecular Cardiology, University \\ of Zurich, Zurich, Switzerland. ${ }^{3}$ Abel Salazar Biomedical Sciences Institute, University of Porto, Porto, Portugal. ${ }^{4}$ Laboratory of Integrative and Systems Physiology, Institute of Bioengineering, School of Life \\ Sciences, École Polytechnique Fédérale de Lausanne, Lausanne, Switzerland. ${ }^{5}$ Center of Animal Biotechnology and Gene Therapy and Department of Biochemistry and Molecular Biology, School of Veterinary \\ Medicine, Universitat Autònoma de Barcelona, Bellaterra, and Centro de Investigación Biomédica en Red de Diabetes y Enfermedades Metabólicas Asociadas (CIBERDEM), Barcelona, Spain. \\ ${ }^{6}$ Department of Pediatrics, Center for Liver Digestive and Metabolic Diseases, University of Groningen, University Medical Center Groningen, Groningen, The Netherlands.
}

\begin{abstract}
Hepatic steatosis is caused by metabolic imbalances that could be explained in part by an increase in de novo lipogenesis that results from increased sterol element binding protein 1 (SREBP-1) activity. The nuclear receptor liver receptor homolog 1 (LRH-1) is an important regulator of intermediary metabolism in the liver, but its role in regulating lipogenesis is not well understood. Here, we have assessed the contribution of LRH-1 SUMOylation to the development of nonalcoholic fatty liver disease (NAFLD). Mice expressing a SUMOylation-defective mutant of LRH-1 (LRH-1 K289R mice) developed NAFLD and early signs of nonalcoholic steatohepatitis (NASH) when challenged with a lipogenic, high-fat, high-sucrose diet. Moreover, we observed that the LRH-1 K289R mutation induced the expression of oxysterol binding protein-like 3 (OSBPL3), enhanced SREBP-1 processing, and promoted de novo lipogenesis. Mechanistically, we demonstrated that ectopic expression of OSBPL3 facilitates SREBP-1 processing in WT mice, while silencing hepatic Osbp/3 reverses the lipogenic phenotype of LRH-1 K289R mice. These findings suggest that compromised SUMOylation of LRH-1 promotes the development of NAFLD under lipogenic conditions through regulation of OSBPL3.
\end{abstract}

\section{Introduction}

Hepatic steatosis is characterized by the excessive accumulation of triglycerides as a consequence of an imbalance between the acquisition and disposal of fatty acids. Increased hepatic uptake of adipose tissue-derived fatty acids, defective breakdown via $\beta$-oxidation, reduced VLDL-triglyceride secretion, and induced activity of master regulators of de novo lipogenesis, such as sterol element binding protein 1 (SREBP-1, encoded by Srebf1), are factors that contribute to the development of steatosis (1). Nonalcoholic fatty liver disease (NAFLD) comprises a spectrum of disorders, of which the earliest stage is characterized by the deposition of lipid droplets within the cytoplasm of the hepatocytes. NAFLD increases the susceptibility to hepatocyte damage and inflammation, a condition termed nonalcoholic steatohepatitis (NASH), and can ultimately progress to cirrhosis and hepatocellular carcinoma (HCC) (1-4).

Liver receptor homolog 1 (LRH-1) is a nuclear receptor with diverse biological functions ranging from cell cycle regulation to the control of steroid homeostasis. In the liver, LRH-1 is an important regulator of glucose, cholesterol, and bile acid metabolism (5). Liver-specific Lrh-1 knockout mice display reduced glycolytic flux and de novo lipogenesis secondary to impaired glucokinase activity

Authorship note: S. Stein and V. Lemos contributed equally to this work. Conflict of interest: The authors have declared that no conflict of interest exists. Submitted: November 11, 2015; Accepted: November 22, 2016.

Reference information: J Clin Invest. 2017;127(2):583-592.

https://doi.org/10.1172/JCl85499.
(6). On the other hand, treatment of mice with the LRH-1 agonist 1,2-dilauroyl-sn-glycero-3-phosphocholine (DLPC) protects animals from developing NAFLD and insulin resistance in genetic and dietary models of diabesity $(7,8)$. To address this apparent contradictory role of LRH-1 in hepatic triglyceride metabolism, we chose to study lipogenesis in our recently described Lrh-1 K289R knockin mouse model (LRH-1 K289R mice), which displays selective gain of function of LRH-1 as a result of impaired SUMOylation at K289 (9).

In this study, we demonstrate that LRH-1 K289R induces the expression of oxysterol binding protein-like 3 (OSBPL3, encoded by Osbpl3, also known as Orp3), which in turn promotes the posttranslational activation of SREBP-1. As a consequence, LRH-1 K289R mice display increased de novo lipogenesis upon refeeding in comparison with WT mice. In fact, compared with what is seen in LRH-1 WT mice, this chronic lipogenic stress promotes NAFLD in LRH-1 K289R mice accompanied by early signs of NASH when mice are exposed to a lipogenic, high-fat, high-sucrose (HFHS) diet.

\section{Results}

Increased hepatic SREBP-1 processing in LRH-1 K289R mice. In order to evaluate the role of an LRH-1 SUMO-defective pathway on intermediary liver metabolism, we subjected LRH-1 K289R mice, which exhibit partial gain of function of LRH-1, and control LRH-1 WT mice (9) to fasting-refeeding challenges in which mice were fasted and then refed for a period of 6 hours. We then evaluated the expression of metabolic genes in refed livers of both genotypes using microarray analysis. Interestingly, the expression of 
A

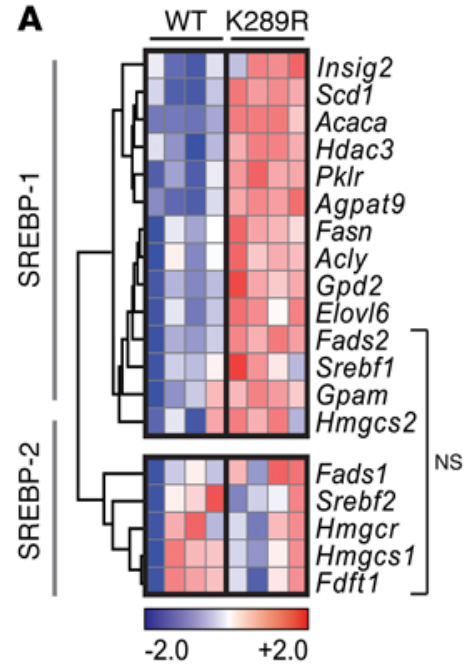

D
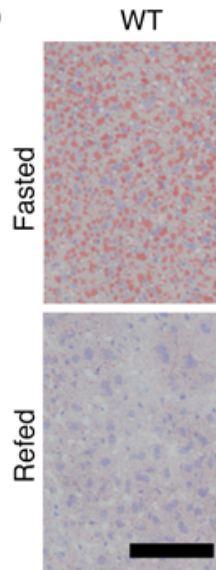

B
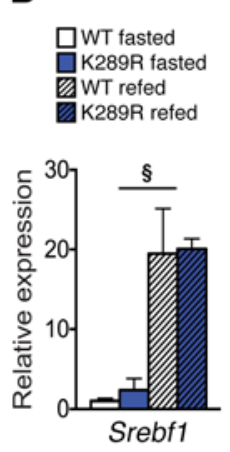

C

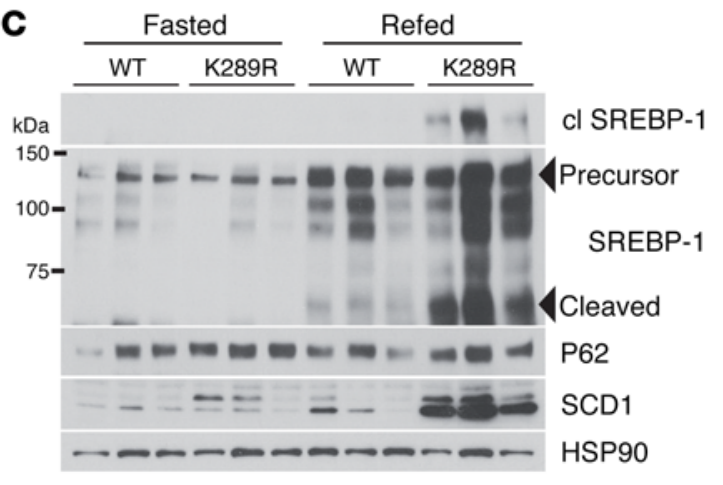

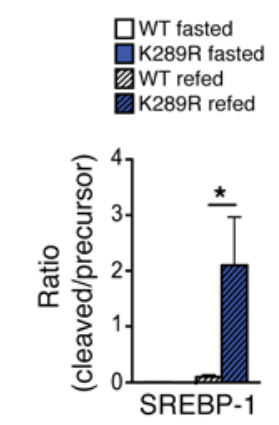

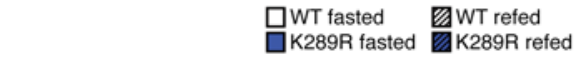

E
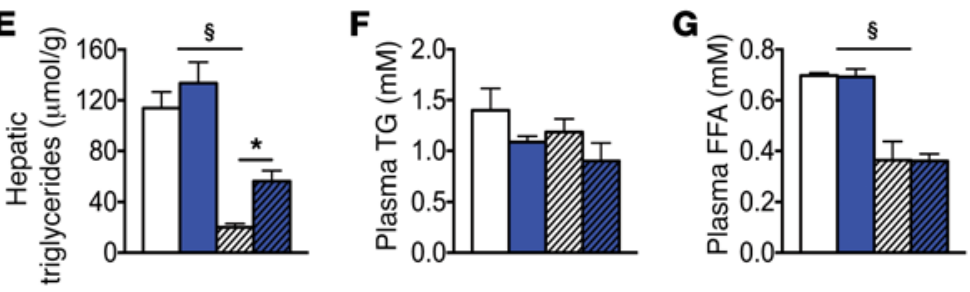

H
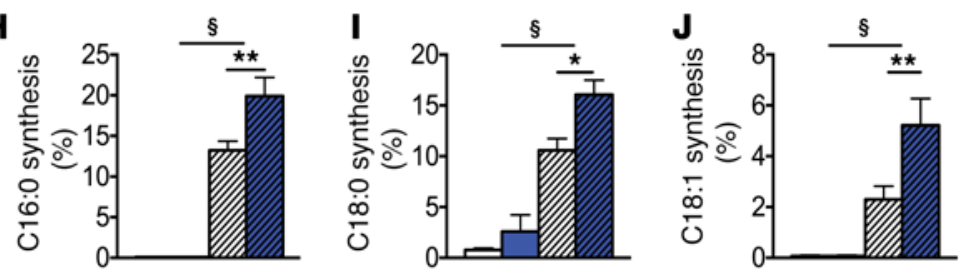

Figure 1. LRH-1 K289R mice display increased de novo lipogenesis. (A) Heat map showing the expression of genes involved in de novo fatty acid and cholesterol synthesis in refed WT and K289R mice. Normalized expression values are in $\log _{2}$ scale. SREBP-1, depicting mainly SREBP-1 target genes; SREBP-2, mostly SREBP-2 target genes; NS, transcripts that are not significantly changed between the indicated genotypes. For all other transcripts $P<0.05$. (B) Hepatic mRNA expression of Srebf1 in K289R and WT mice. $n=10$ per genotype. (C) Left, immunoblots of precursor and cleaved (cl) SREBP-1, SCD1, HSP90, and P62 in hepatic lysates of WT or K289R livers. Right, graph displaying the ratio of cleaved to precursor SREBP-1. (D) Representative images of liver sections of K289R or WT mice stained with oil red 0 to visualize neutral lipids. Scale bar: $200 \mu \mathrm{m}$. (E) Quantification of hepatic triglyceride content in WT and K289R mice. $n=10$ per group. (F and $\mathbf{G}$ ) Plasma triglyceride (TC) and free fatty acid (FFA) contents in WT and K289R mice. $n=10$ per group. (H-J) Fractional de novo synthesis rates of palmitate $(\mathbf{H})$, stearate $(\mathbf{I})$, and oleate $(\mathrm{J})$ in WT and K289R mice. $n=6$ per group. Error bars represent mean \pm SEM. ${ }^{*} P<0.05,{ }^{* *} P<0.01$ relative to WT within each nutritional state; ${ }^{\S} P<0.001$ refed relative to fasted mice, as determined by unpaired Student's $t$ test (A) or 2-way ANOVA with Bonferroni's post-hoc test (B, C, E-J). WT, LRH-1 WT; K289R, LRH-1 K289R mice.

many SREBP-1 target genes was increased in 6-hour-refed LRH-1 K289R compared with LRH-1 WT livers, whereas SREBP-2 targets were not altered (Figure 1A). Although no changes in Srebfl mRNA (Figure 1B) or uncleaved precursor SREBP-1 (Figure 1C) could be observed between both genotypes, refed LRH-1 K289R livers displayed significantly more of the cleaved and transcriptionally active SREBP-1 protein in comparison with the LRH-1 WT livers (Figure 1C), indicating that the posttranslational processing and maturation of SREBP-1 is increased in LRH-1 K289R mice. We then performed acute insulin challenges as well as shorter 2-hour refeeding experiments to analyze whether early signaling events could explain the increased SREBP-1 activity. Insulin did not induce an additional increase in AKT phosphorylation or a consistent induction of early response genes, such as activating transcription factor 3 (Atf3) or early growth response 1 (Egr1) (Supple- mental Figure 1, A and B; supplemental material available online with this article; https://doi.org/10.1172/JCI85499DS1) (10, 11), in LRH-1 K289R versus LRH-1 WT livers. Similarly, we did not observe differences in Srebfla and Srebflc expression in 2-, 6-, or 12-hour-refed LRH-1 K289R and LRH-1 WT mice (Supplemental Figure 1C). Taken together, these data suggest that LRH-1 K289R induces SREBP-1 signaling primarily at the posttranscriptional level independently of early insulin-AKT signaling.

Enhanced de novo lipogenesis in LRH-1 K289R mice. To verify whether the increased expression of lipogenic genes is accompanied by hepatic fat accumulation, we next quantified hepatic triglyceride content in LRH-1 K289R and LRH-1 WT mice. The content of neutral lipids and triglycerides was significantly increased in LRH-1 K289R compared with LRH-1 WT livers upon refeeding (Figure 1, D and E). Of note, plasma triglycerides and free fatty 
A

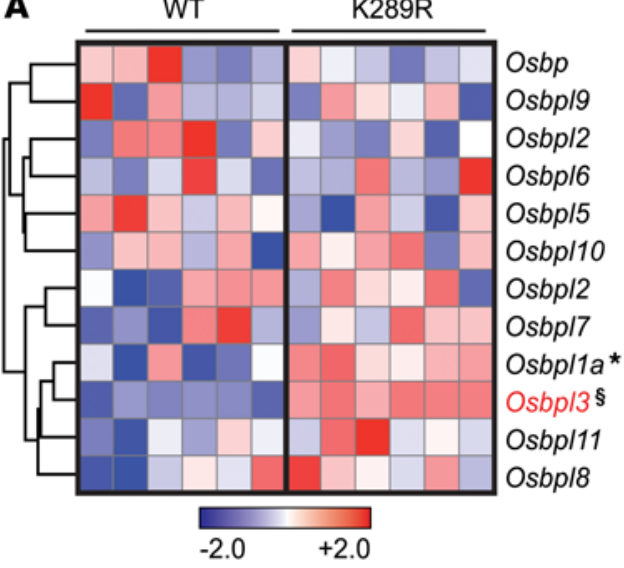

B
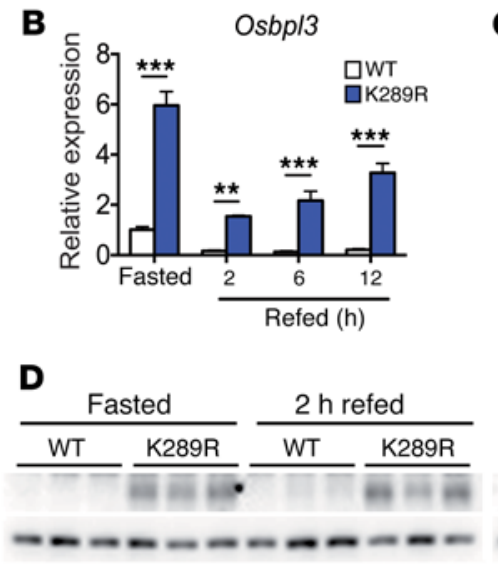
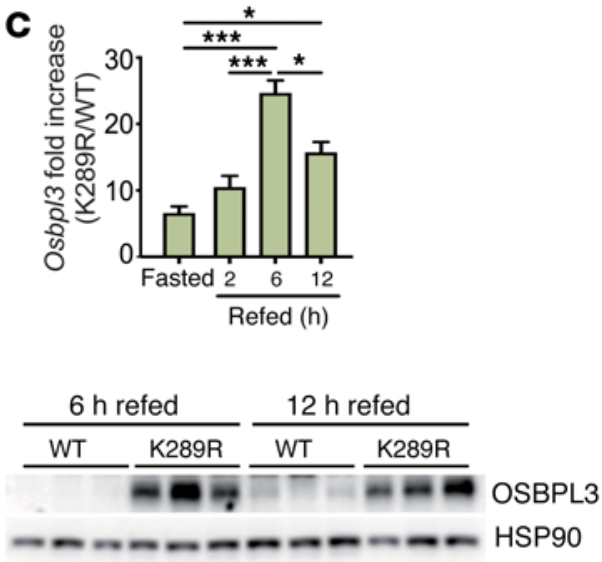

$\mathbf{E}$

Osbp/3 genomic region
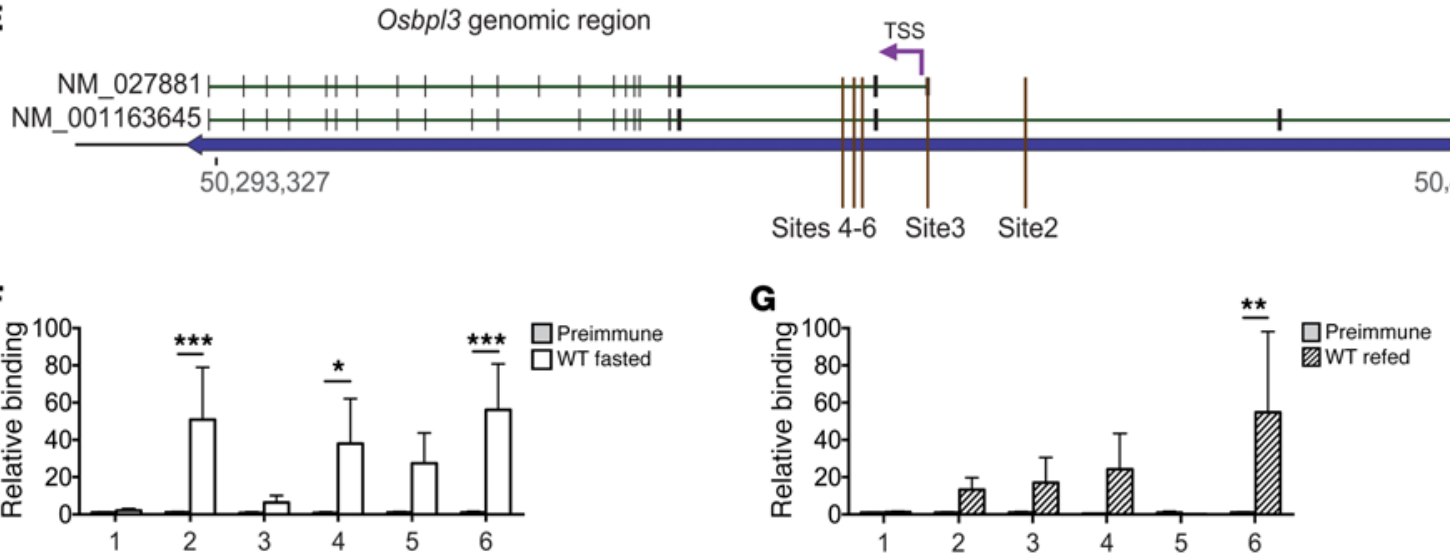

Figure 2. Osbpl3 is a direct LRH-1 target gene and overexpressed in LRH-1 K289R mice. (A) Heat map showing the hepatic expression of oxysterol-binding protein family members in WT and K289R mice. Normalized expression values are in $\log _{2}$ scale. (B and C) Expression of Osbp/3 mRNA in hepatic lysates of fasted and refed WT and K289R mice (B) and the fold change between the genotypes (C). $n=4$ per fasted, 2-hour-, or 12-hour-refed groups, and $n=5$ per 6-hour-refed groups. (D) Expression levels of OSBPL3 protein in hepatic lysates of fasted and refed WT and K289R mice. (E) Schematic showing the genomic area containing the $0 \mathrm{sbpl} 3$ gene and the sites used for ChIP-qPCR experiments (mouse genome assembly mm10). (F and $\mathbf{G})$ Binding of LRH-1 to the different Osbp/3 promoter sites assessed by ChIP analysis using genomic DNA from fasted WT (F) and refed WT livers (G). $n=5$ WT fasted, $n=5$ WT refed. Error bars represent mean $\pm \mathrm{SEM}$. ${ }^{*} P<0.05,{ }^{* *} P<0.01,{ }^{* *} P<0.001,{ }^{\circledR} P=2 \times 10^{-7}$ relative to WT, as determined by unpaired Student's $t$ test (A), or 1-way (C) or 2-way (B, F, G) ANOVA with Bonferroni post-hoc test. TSS, transcription start site.

acids did not show significant alterations between LRH-1 K289R and LRH-1 WT mice (Figure 1, F and G). To verify whether the enhanced expression of lipogenic genes translates into increased de novo lipogenesis, animals received ${ }^{13} \mathrm{C}$-acetate prior to sacrifice to quantify de novo lipogenesis (6). In line with the increased lipogenic gene expression, de novo synthesis of palmitate (C16:0), stearate (C18:0), and oleate (C18:1) was significantly higher in LRH-1 K289R compared with LRH-1 WT livers (Figure 1, H-J). Of note, chain elongation of preexisting fatty acids was not altered between the 2 genotypes (Supplemental Figure 1, D and E). Collectively, these data show that LRH-1 K289R mice display increased de novo lipogenesis and that enhanced activation of SREBP-1 likely contributes to this process.

SUMOylation-defective $L R H-1$ drives the expression of Osbpl3. We next analyzed the transcriptome of livers from refed LRH-1 K289R and LRH-1 WT mice and searched for transcripts that are linked to SREBP-1 processing. One of the top hits on the list of genes that was increased in LRH-1 K289R compared with LRH-1 WT livers corresponded to $O s b p l 3$, a member of a class of lipid transfer proteins recently implicated in the shuttling of lipids between the plasma and endoplasmic reticulum membrane (12-14). The expression of Osbpl3, and to a lesser extent Osbpl1a, was robustly induced in LRH-1 K289R livers, while that of most other OSBP family members did not differ between the 2 genotypes (Figure 2A). Of interest, overexpression of certain oxysterol-binding proteins has been suggested as increasing SREBP-1 processing and hepatic lipogenesis (15). Analysis of livers of LRH-1 K289R versus LRH-1 WT mice confirmed the enhanced expression of Osbpl3 mRNA during fasting and refeeding (Figure 2B), while the expression of other OSBP family members did not differ between the 2 genotypes (Supplemental Figure 2A). Notably, the expression of Osbpl3 was high during fasting and then reduced upon refeeding (Figure $2 \mathrm{~B}$ ). This postprandial suppression of Osbpl3 mRNA expression was also observed upon overexpression of Osbpl3 in mice using an adenovirus (Supplemental Figure 2B), suggesting that during the refed state, Osbpl3 is regulated by posttranscriptional mechanisms occurring independently of LRH-1. Despite this feeding-dependent regulation of the mRNA, hepatic Osbpl3 mRNA levels were consistently higher in the LRH-1 K289R 
A Fasted B

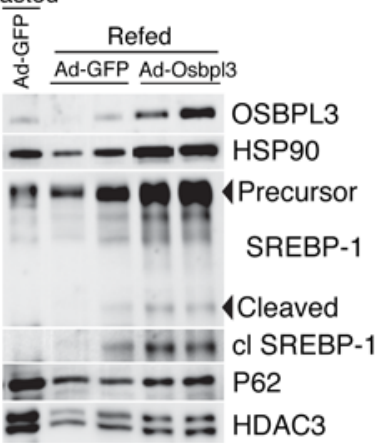

E

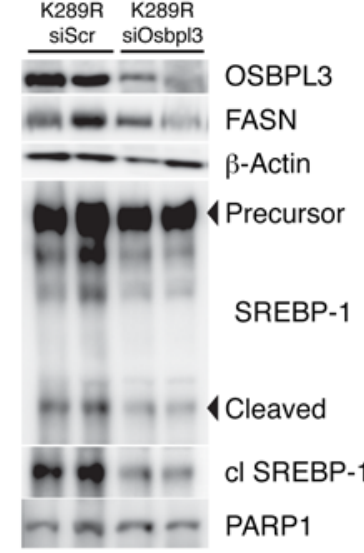

B

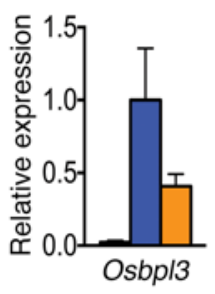

C

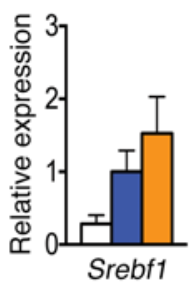

D

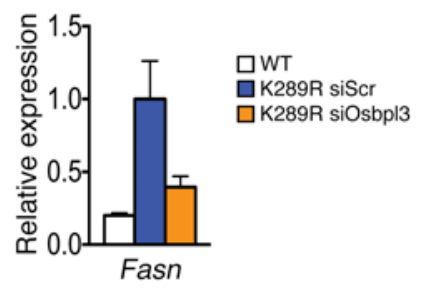

$\mathbf{F}$

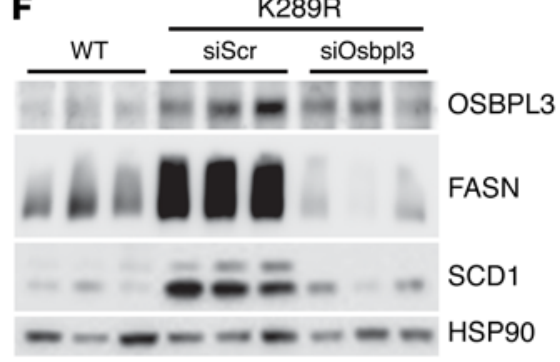

I

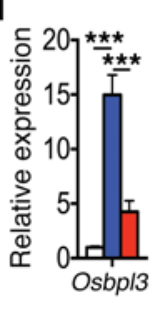

J

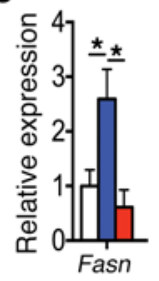

G

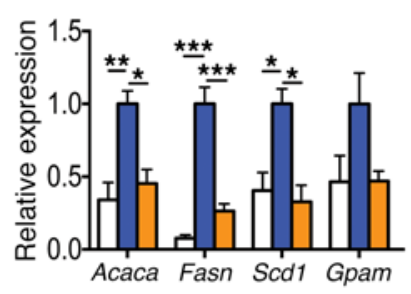

H

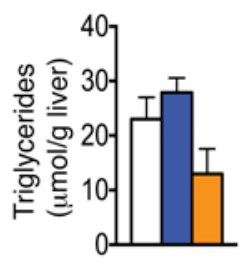

$\mathbf{K}$

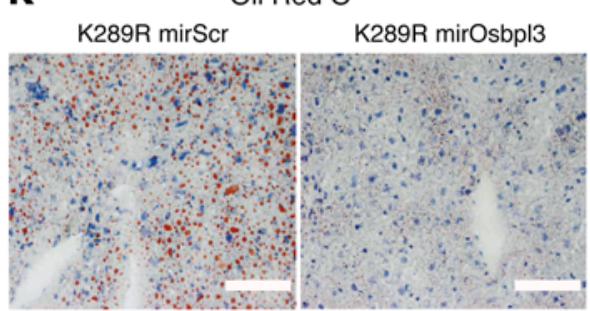

Figure 3. Osbp/3 silencing rescues the lipogenic phenotype of LRH-1 K289R mice. (A) Immunoblots of OSBPL3, HSP90, precursor and cleaved SREBP-1, P62, and HDAC3 in hepatic lysates of fasted or refed WT plus Ad-GFP or refed WT plus Ad-OSBPL3 livers. (B-D) Hepatic mRNA expression of Osbpl3 (B), Srebf1 (C), and Fasn (D) in refed K289R and WT mice. WT, $n=3$; K289R siScr or K289R siOsbpl3, $n=9$ per genotype. (E) Immunoblots of OSBPL3, FASN, $\beta$-actin, precursor and cleaved SREBP-1, and PARP1 in hepatic lysates of refed K289R siScr or K289R siOsbpl3 livers. (F) Hepatic expression of OSBPL3, FASN, SCD1, and HSP9O in livers of ad libitum-fed WT, K289R siScr, and K289R siOsbpl3 mice. (C) Hepatic mRNA expression of genes involved in de novo lipogenesis in ad libitum-fed WT, K289R siScr, and K289R siOsbpl3 mice. $n=3$ per genotype. (H) Quantification of hepatic triglycerides in hepatic lipid extracts from ad libitum-fed WT, K289R siScr, and K289R siOsbpl3 mice. $n=3$ per genotype. (I and J) Hepatic mRNA expression of Osbp/3 (I) and Fasn (J) in 6-hour-refed WT, K289R, or K289R mirOsbpl3 mice. $n$ = 3 per genotype. (K) Representative oil red 0 staining in liver cryosections from refed K289R mirScrambled or K289R mirOsbpl3 mice. Scale bars: $100 \mu \mathrm{m}$. Error bars represent mean $\pm \mathrm{SEM} .{ }^{*} P<0.05,{ }^{* *} P<0.01,{ }^{* * *} P<0.001$ relative to K289R siScr, as determined by 1-way ANOVA with Bonferroni's post-hoc test (B-D, G-J). WT, LRH-1 WT; K289R siOsbpl3, LRH-1 K289R mice injected with Osbpl3siRNA complexes; K289R siScr, LRH-1 K289R mice injected with scrambled-siRNA complexes; K289R mirOsbpl3, LRH-1 K289R mice injected with AAV8 viral vectors containing an miRNA targeting Osbpl3.

mice in all nutritional states, but were particularly high under 6-hour-refeeding conditions (Figure 2C), and these differences were translated into similar changes at the protein level (Figure 2D).

To investigate whether Osbpl3 is directly controlled by LRH-1, we first analyzed the Osbpl3 genomic region in available ChIP-Seq data for potential LRH-1-binding sites $(16,17)$. We identified 3 main sites from the ChIP-Seq data from Holmstrom et al. (sites 1-3; ref. 16), and 3 additional sites with an LRH-1 consensus sequence close to one of the transcription start sites of the Osbpl3 gene (sites 4-6) were identified by computational analysis (Figure 2E). We then performed site-specific ChIP analysis to evaluate whether these sites are bound by LRH- 1 in fasted and refed mice. LRH-1 was recruited at different sites under both nutritional conditions (Figure 2, F and G), suggesting that Osbpl3 is a direct LRH-1 target gene.

Based on the fact that LRH-1 and the nuclear receptor liver X receptor (LXR) have been shown to crosstalk in the regulation of hepatic acute-phase response proteins $(18,19)$ and that various
LRH-1 target genes, including lipogenic genes, are coregulated by members of the LXR subfamily (20-22), we assessed the possibility that the LRH-1 mutant may modulate the recruitment of LXR $\alpha$ to its target genes, Abca1, Chrebp, and Srebfl. Although recruitment of LXR $\alpha$ was detected on these promoters in the genomic lysates of LRH-1 K289R and LRH-1 WT livers, no differences were observed between both genotypes, suggesting that LXR $\alpha$ binding is not altered due to the LRH-1 K289R mutation (Supplemental Figure 3A). Furthermore, Hepa 1.6 and AML-12 cells treated with the LXR agonist GW3965 did not alter Osbpl3 expression (Supplemental Figure 3, B and C), indicating that Osbpl3 expression is not regulated by LXR.

Osbpl3 silencing rescues the steatotic phenotype of LRH-1 K289R mice. To establish whether the increased expression of OSBPL3 in LRH-1 K289R livers is causatively linked to exacerbated de novo lipogenesis, we performed in vivo overexpression and silencing experiments. Adenoviral overexpression of OSBPL3 increased 
A

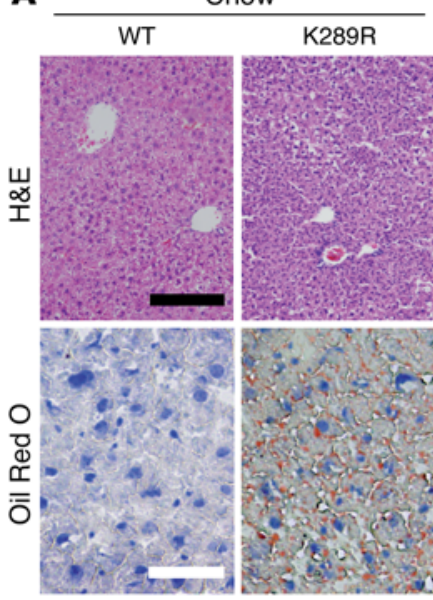

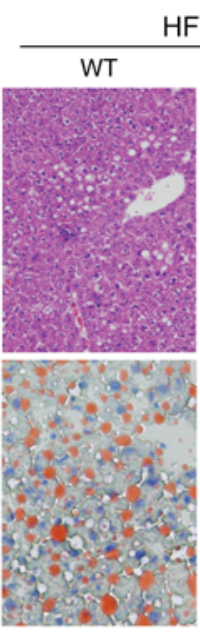

FHS

K289R

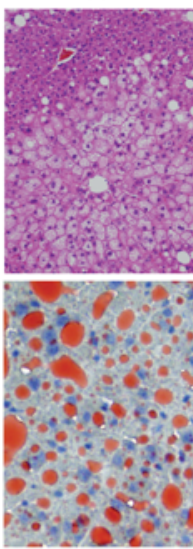

B
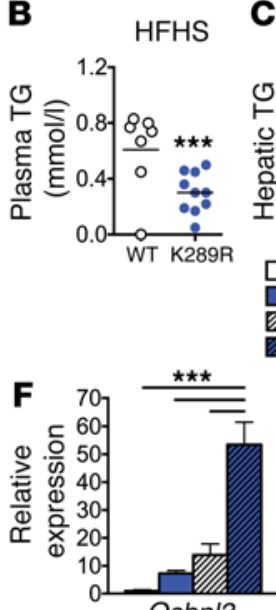

C

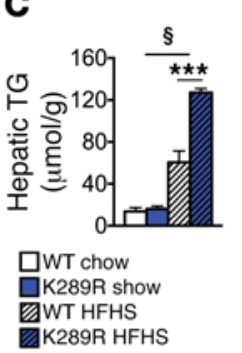

D HFHS E

E HFHS

H

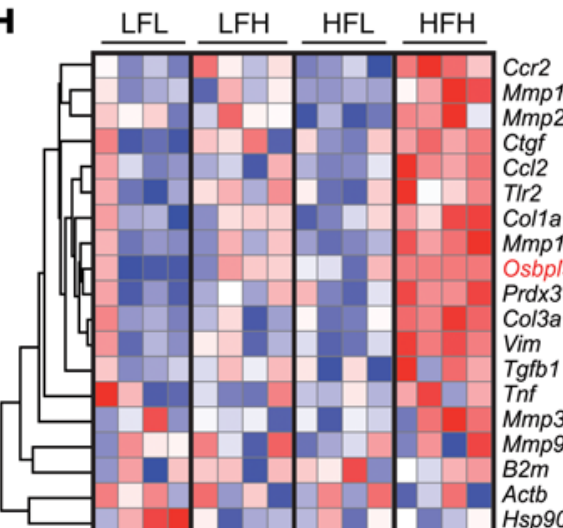

I

Mild NAFLD

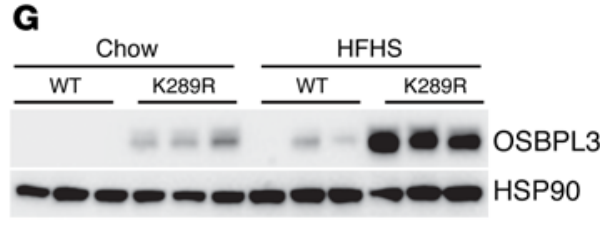

Mmp13

Mmp2

Ctgf

$\mathrm{Ccl} 2$

Tlr2

Mmp12

Sbp/3

Col3a1

Vim

Tgfb1

inf

Mmp9

B2m

Hsp90b1

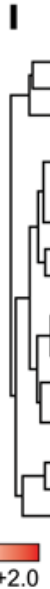

Advanced NAFLD

$$
-2.0+2.0
$$

Figure 4. LRH-1 K289R mice develop NAFLD upon HFHS diet feeding. (A) Representative images of liver sections of K289R or WT mice stained with H\&E or oil red 0 to visualize the tissue structure and neutral lipids, respectively. Black scale bar: $200 \mu \mathrm{m}$; white scale bar: $50 \mu \mathrm{m}$. (B and C) Quantification of triglyceride content in plasma (B) and in hepatic lipid extracts (C) in WT and K289R mice. WT, $n=7$; K289R, $n=10$. (D and E) Plasma levels of ALAT (D) and ASAT (E) in mice fed a HFHS diet. WT, $n=7 ; \mathrm{K} 289 \mathrm{R}, n=10$. (F and G) Expression of Osbp/3 mRNA (F) and protein (G) levels in livers of WT and K289R mice fed chow and HFHS diets. $n=9$ per genotype. (H) Heat map displaying the expression of Osbpl3 as well as markers of matrix degradation, fibrosis, and inflammation in mice that were classified as LFL responders, LFH responders, HFL responders, and HFH responders according to the development of NAFLD/NASH upon chow or high-fat diet feeding (24). (I) Expression of OSBPL3 and markers of matrix degradation, fibrosis, and inflammation in

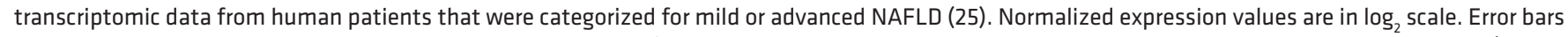
represent mean \pm SEM. ${ }^{* *} P<0.01$, ${ }^{* *} P<0.001$ relative to WT; ${ }^{\circledR} P<0.001$ refed relative to fasted mice, as determined by unpaired Student's $t$ test $(\mathbf{B}$, $\mathbf{D}$, E) or 2-way ANOVA with Bonferroni's post-hoc test (C, F).

SREBP-1 cleavage in refed LRH-1 WT mice (Figure 3A), suggesting that elevated OSBPL3 levels promote SREBP-1 activation. We next silenced Osbpl3 in LRH-1 K289R mice using siRNAs in LRH-1 K289R mice under fast-refeeding conditions. The hepatic mRNA expression of Osbpl3 showed a clear trend of effective silencing (Figure 3B), while Srebf1 expression was not altered (Figure 3C). Importantly, we observed a robust decrease in OSBPL3 protein along with a reduction in the maturation of SREBP- 1 and a blunted expression of the fatty acid synthase (FASN) transcript and protein in the livers of refed LRH-1 K289R mice treated with siOsbpl3 (Figure 3, D and E). We next assessed the effect of Osbpl3 silencing under normal-fed conditions. Western blot analysis revealed reduced OSBPL3 expression (Figure 3F) and a robust decrease in the lipogenic proteins FASN and stearoyl-coenzyme A desaturase 1 (SCD-1) in ad libitum-fed LRH-1 K289R mice treated with siOsbpl3 compared with control mice (Figure 3F). Of interest, mRNA expression levels of the lipogenic enzymes acetyl-coenzyme A carboxylase alpha (Acaca), Fasn, Scd1, and glycerol-3-phosphate acyltransferase (Gpam) were often normalized to values observed in LRH-1 WT mice (Figure 3G), suggesting that Osbpl3 silencing rescues the lipogenic phenotype of LRH-1 K289R mice. In line with the reduced expression of lipogenic genes, Osbpl3 silencing also decreased hepatic triglyceride and neutral lipid content (Figure $3 \mathrm{H})$. As an additional approach to silence Osbpl3, we injected mice with an AAV8 viral vector containing an miRNA targeting Osbpl3 in the liver. Here again, we observed a robust silencing of Osbpl3, which was accompanied by the suppression of Fasn and a striking reduction of hepatic lipid droplets (Figure 3, I-K).

Finally, we also examined whether other mechanisms, directly or indirectly regulated by LRH-1, may contribute to the lipogenic phenotype. Of interest, both hepatic glucose-6-phosphate content (6) and VLDL secretion (23) were unchanged between 
A

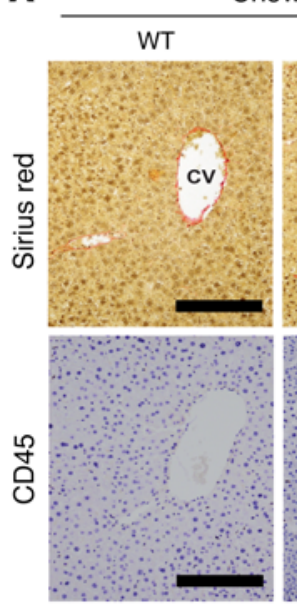

B

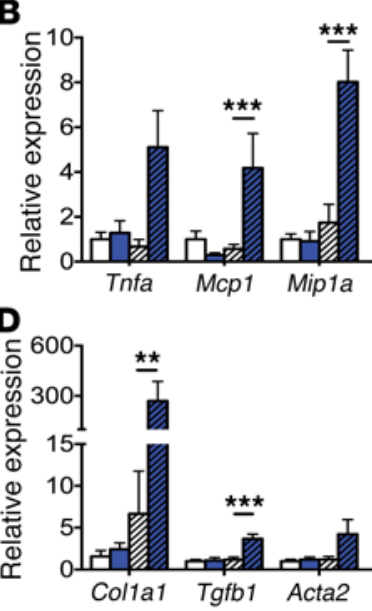

K289R
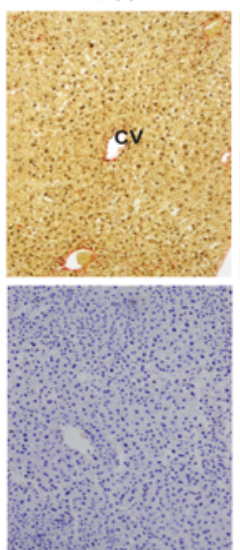

C

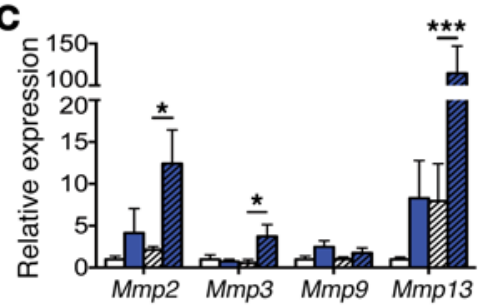

E

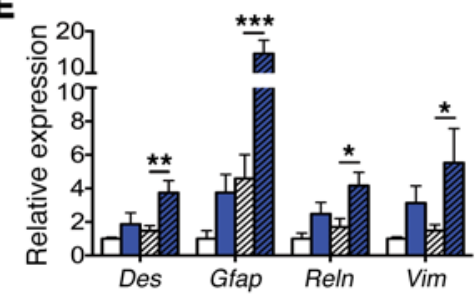

$\mathbf{F}$
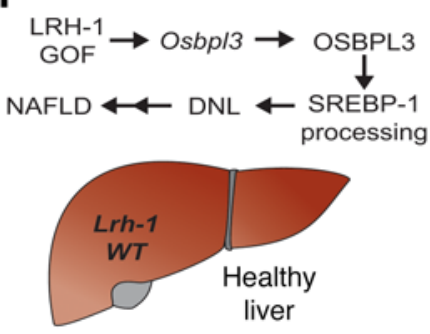

- Normal LRH-1 activity

- Low OSBPL3 expression

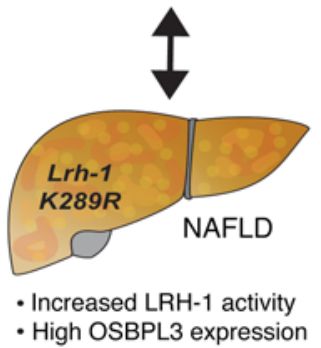

Figure 5. LRH-1 K289R mice display increased inflammation and early signs of fibrosis upon HFHS diet feeding. (A) Representative images of liver sections of K289R or WT mice stained with sirius red or CD45 to visualize collagen depositions and CD45-positive cells, respectively. Scale bars: $200 \mu \mathrm{m}$. CV, central vein. (B-E) Hepatic mRNA expression of genes involved in inflammation (B), matrix degradation (C), fibrosis (D), and stellate cells (E) in K289R and WT mice. $n=9$ per genotype. (F) Graphical presentation showing how the LRH-1/OSBPL3 axis drives the accumulation of hepatic lipids. Error bars represent mean \pm SEM. ${ }^{*} P<0.05,{ }^{* *} P<0.01,{ }^{* *} P<0.001$ relative to WT within each diet, as determined by 2-way ANOVA with Bonferroni's post-hoc test (B-E).

LRH-1 K289R and LRH-1 WT mice (Supplemental Figure 4, A-C), making it unlikely that these processes account for the exacerbated lipid accumulation in liver.

Together, these data suggest that the LRH-1-OSBPL3 signaling axis contributes to the increased maturation of SREBP-1, the induction of lipogenic enzymes, and the hepatic accumulation of triglycerides observed in LRH-1 K289R mice.

LRH-1 K289R mice are prone to developing NAFLD upon lipogenic diet feeding. Excessive de novo lipogenesis contributes to the development of NAFLD, a condition that can eventually progress to NASH $(1,3)$. In order to investigate whether LRH-1 affects the development of NAFLD, LRH-1 K289R and LRH-1 WT mice were fed a highly lipogenic HFHS diet or a chow diet for 17 weeks, and then sacrificed under normal-fed conditions. Stainings of hepatic sections with $\mathrm{H} \& \mathrm{E}$ and oil red O revealed that LRH-1 K289R mice developed a stronger steatotic phenotype compared with LRH-1 WT mice fed a HFHS diet (Figure 4A). While plasma cholesterol levels were unchanged (Supplemental Figure 5A), triglyceride content in plasma (Figure 4B) or in VLDL fractions (Supplemen- tal Figure 5B) was reduced in HFHS-fed LRH-1 K289R mice. This reduction was accompanied with a robust accumulation of hepatic triglycerides (Figure 4C) as well as an induction of plasma levels of the enzymes alanine transaminase (ALAT) and aspartate aminotransferase (ASAT) in the HFHS-challenged LRH-1 K289R mice (Figure 4, D and E). Similar to our earlier observations in normal chow diet conditions (Figure 2, A-D), Osbpl3 mRNA and OSBPL3 protein were significantly induced in LRH-1 K289R compared with LRH-1 WT mice fed a HFHS diet (Figure 4, F and G), while the expression of other Osbp genes did not differ between the genotypes (Supplemental Figure 5C).

The development of hepatic steatosis in mice is known to be highly heterogeneous (24). Therefore, we analyzed the data from a study in which mice were fed a chow or high-fat diet and then classified according to the development of NAFLD/NASH into lowfat low (LFL) responders, low-fat high (LFH) responders, high-fat low (HFL) responders, and high-fat high (HFH) responders (24). Interestingly, the expression of Osbpl3 was significantly induced in the $\mathrm{HFH}$ responders along with markers of inflammation and 
necrosis (Figure $4 \mathrm{H}$ ) and was by far the most strikingly increased Osbp among all family members (Supplemental Figure 5D). We also analyzed OSBPL3 expression in 2 cohorts of NAFLD/NASH patients. In the first cohort, livers were categorized as showing mild or advanced NAFLD (25). In the second study, subjects had livers ranging from healthy controls to showing steatosis and further to NASH (26). OSBPL3 expression was low in healthy livers, but increased in advanced stages of NAFLD/NASH and clustered with markers of fibrosis (Figure 4I and Supplemental Figure 6), suggesting that OSBPL3 could be a novel biomarker for advanced liver diseases, such as NASH. Taken together, these data show that Osbpl3 is markedly increased in LRH-1 K289R mice as well as in mice and humans with NAFLD/NASH.

LRH-1 K289R mice display increased inflammation and early signs of fibrosis in response to lipogenic diet feeding. Given the strong steatotic phenotype and the high levels of ALAT and ASAT markers in LRH-1 K289R mice fed a HFHS diet as well as the clustering of Osbpl3 with genes involved in inflammation and fibrosis in mice and humans with NAFLD, we next analyzed inflammatory and fibrotic markers in LRH-1 K289R and LRH-1 WT mice. Stainings of hepatic sections with sirius red and CD45 revealed that, in contrast with LRH-1 WT mice, LRH-1 K289R mice developed small fibrotic lesions and accumulated CD45-positive immune cells upon HFHS feeding (Figure 5A). Moreover, LRH-1 K289R livers displayed higher levels of the inflammatory genes Tnfa, monocyte chemoattractant protein-1 ( $M c p 1$ or Ccl2), and macrophage inflammatory protein-1 $\alpha$ (Mip1 $\alpha$ or Ccl3) (Figure $5 \mathrm{~B})$, indicating increased inflammation in livers of HFHS-fed LRH-1 K289R mice. The expression of MMPs, such as Mmp2 and $M m p 13$, as well as early markers of fibrosis, such as $\alpha-1$ type I collagen (Col1a1) and Tgfb1, was also elevated in LRH-1 K289R in comparison with LRH-1 WT mice fed a HFHS diet (Figure 5, C and D). Hepatic stellate cells are the primary hepatic cell type promoting fibrogenesis (27). The expression of different hepatic stellate cell markers, such as desmin (Des), glial fibrillary acidic protein (Gfap), and reelin (Reln), was also increased in HFHSfed LRH-1 K289R compared with LRH-1 WT mice (Figure 5E), suggesting that HFHS-treated LRH-1 K289R mice exhibit early signs of NASH.

Previous studies showed that LRH-1 and the SUMOylation machinery might affect the hepatic acute phase response (APR) $(18,19,28)$. While induction of the APR by short exposure of LPS led to reduced hepatic expression of Crp and Tnf in LRH-1 K289R compared with LRH-1 WT mice, it did not alter the hepatic expression of other APR and inflammatory genes (Supplemental Figure 7A). Moreover, no difference in plasma IL-6, MCP-1 (also known as CCL-2), or TNF- $\alpha$ could be observed in LPS-challenged LRH-1 K289R and LRH-1 WT mice (Supplemental Figure 7B), suggesting that the APR is likely not driving the observed inflammatory phenotype in our NAFLD-model.

Taken together, these data show that SUMOylation-defective LRH-1 promotes the development of NAFLD and displays early signs of NASH in mice fed a HFHS diet. This process is at least partially driven by the LRH-1-OSBPL3 signaling axis, which contributes to increased maturation of SREBP-1, the induction of lipogenic enzyme expression, and hepatic triglyceride accumulation in LRH-1 K289R mice (Figure 5F).

\section{Discussion}

In this study, we analyzed the contribution of a selective LRH-1 gain of function on hepatic de novo lipogenesis and fatty liver development and identified Osbpl3 as a critical component in the regulation of this process. While the hepatic expression of Osbpl3 is normally low in mice under basal conditions, LRH-1 K289R mice express high levels of this specific OSBP family member. Consistent with our observations, the analyses of independent mouse and human transcriptomic data sets revealed that Osbpl3 expression is often induced in NAFLD/NASH. Our work furthermore strengthens the putative role of OSBPL3 in SREBP-1 maturation. As a consequence, LRH-1 K289R mice display increased de novo lipogenesis and accumulation of triglycerides upon refeeding in a manner that is independent of enhanced $\mathrm{LXR} \alpha$ recruitment or activity. Importantly, silencing of OSBPL3 in vivo reverts the increased lipogenesis observed in these animals. Moreover, when exposed to a lipogenic HFHS diet, LRH-1 K289R mice develop NAFLD accompanied by early signs of NASH, most likely as a consequence of chronic fat accumulation $(1,3)$.

The excessive accumulation of hepatic lipids and increased de novo lipogenesis could also have other causes. A previous study using liver-specific Lrh-1 knockout mice showed that LRH-1 promotes glucokinase expression, hence regulating glycolysis and de novo lipogenesis (6). However, we did not observe a difference in hepatic glucose-6-phosphate content between LRH-1 WT and LRH-1 K289R mice, suggesting that the selective gain of function of LRH-1 driven by the K289R mutation does not increase substrate availability for the glycolytic pathway. Based on the finding that the primary corepressor of LRH-1 affects microsomal triglyceride transfer protein levels and consequently hepatic VLDL-triglyceride secretion (23), we also performed in vivo VLDL-secretion assays, but could not detect any difference between refed LRH-1 K289R and LRH-1 WT mice. Finally, LRH-1 SUMOylation has been linked to the induction of the hepatic $\operatorname{APR}(18,19,28)$, which could explain the excessive inflammation observed in the livers of mice receiving a chronic HFHS diet. To study the APR in vivo, we challenged LRH-1 WT and LRH-1 K289R mice with lipopolysaccharide for 2.5 hours. Besides the reduction in Crp and Tnf, the inflammatory signature was comparable between the genotypes, suggesting that the exacerbated inflammatory phenotype of the LRH-1 K289R mice is most likely not driven by changes in the APR.

While the induction of SREBP-2 processing has been well described, the mechanisms that trigger SREBP-1 processing are less well understood (29). Insulin receptor/AKT/mTOR signaling is considered one of the main pathways triggering SREBP-1 signaling (30). A recent study showed that insulin promotes SREBP-1 activation and de novo lipogenesis via mTORC1-dependent and -independent mechanisms (31). Others proposed that while insulin signaling is required for SREBP-1 activation in insulinresistant conditions, it is not critical for inducing the feedingdependent induction of SREBP-1 under physiological conditions $(32,33)$. In breast epithelial cells, the expression of oncogenic forms of the PI3K or K-Ras are sufficient to induce SREBP-1 maturation and de novo lipogenesis through induction of mTOR signaling (34). Whereas these and many other studies demonstrate that the expression of Srebf 1 and the posttranslational maturation of SREBP-1 can be triggered by insulin signaling, other downstream 
or parallel pathways exist. One recent example is the induction of SREBP-1 processing upon depletion of phosphatidylcholine, whose synthesis is dependent on S-adenosylmethionine, a methionine derivative that is generated in the one-carbon cycle (35). We did not observe a difference in AKT phosphorylation between LRH-1 WT and LRH-1 K289R mice, suggesting other signaling pathways to induce the processing of SREBP-1. Indeed, our current study highlights the existence of an alternative mechanism of SREBP-1 activation involving a SUMO-dependent LRH-1/OSBPL3 pathway.

The first oxysterol-binding proteins were identified, purified, and cloned in the 1980s (36-38). Recent studies suggest that OSBPs act as sterol transfer and/or sensor proteins that may also play important roles in cell signaling $(39,40)$. OSBPL3, a member of subfamily III, contains a conserved sterol-binding OSBP homology domain (OHD) as well as a phosphatidylinositol lipid species-binding pleckstrin homology (PH) domain and two 2-phenylalanines in an acidic tract (FFAT) domains that bind to the vesicle-associated membrane protein-associated (VAMP-associated) protein (VAP) $(39,41,42)$. Recently, it has been shown that this last interaction can activate R-RAS, thus reorganizing the actin cytoskeleton and affecting cell polarity and cell-cell adhesion (43). Future studies will be necessary to dissect the exact mechanisms by which OSBPL3 modulates SREBP-1 processing, but its association with the ER could indicate a possible site of regulation.

In addition to the identification of OSBPL3 as a target of LRH-1, our study also highlights the existence of complex regulatory mechanisms to which OSBPL3 is subjected. We observed a discrepancy between mRNA and protein levels of OSBPL3 between fasting and refed conditions despite its persistent increase in LRH-1 K289R mice. The marked reduction in mRNA levels upon refeeding was also noticed when OSBPL3 was overexpressed using an adenovirus, indicating that additional posttranscriptional mechanisms independent of LRH-1 regulate the expression of OSBPL3. Moreover, while the expression of OSBPL3 is low in healthy livers, it is increased in NAFLD, suggesting that this protein could be a novel biomarker for NAFLD.

In this study, we described a function of LRH-1 in the development of fatty liver disease. It is noteworthy that other nuclear receptors also contribute to the development of NAFLD by affecting steatosis, glucose homeostasis, inflammation, and/or fibrosis in the liver. For instance, while activation of LXRs or pregnane $\mathrm{x}$ receptor (PXR) promotes lipogenesis and the development of NAFLD, activation of other nuclear receptors such as PPARs or farnesoid X-activated receptor (FXR) has opposite effects (reviewed in ref. 44). Clinical trials performed in NASH patients using PPAR $\gamma$ or FXR agonists showed that the beneficial effects observed on hepatic steatosis and inflammation were accompanied by adverse effects on obesity and hypercholesterolemia, respectively $(45,46)$, highlighting the functional complexity of nuclear receptors (44). Therefore, the development of drugs targeting specific nuclear receptor targets, such as OSBPL3, might provide alternative therapeutic options for treating NAFLD.

\section{Methods}

Animal studies. Congenic C57BL/6J LRH-1 WT or LRH-1 K289R (9) as well as C57BL/6J Lrh-1 $1^{\text {hep-/- }}$ and Lrh- $1^{\text {hep+/t+ }}$ mice (6) were kept under normal housing conditions. For fast-refeeding protocols, 12- to 16-week- old male mice were initially fasted for 24 hours, followed by refeeding for 12 hours ( 8 pm to $8 \mathrm{am}$ ), 6 hours ( 2 am to $8 \mathrm{am}$ ), or 2 hours (6 am to $8 \mathrm{am}$ ), and finally sacrificed together at $8 \mathrm{am}$ to avoid confounding effects of the circadian rhythm. To induce a chronic hepatic steatosis, male mice were fed ad libitum with a HFHS diet (TD.08811, Harlan Laboratories) for 17 weeks and sacrificed at $9 \mathrm{am}$. To study the APR, male mice fed a chow diet received an intraperitoneal injection of either $500 \mu \mathrm{l}$ PBS or $40 \mu \mathrm{g}$ LPS dissolved in $500 \mu \mathrm{l}$ PBS. Mice were sacrificed 2.5 hours after injection and tissues collected.

Subcellular fractionation of liver tissues and Western blotting. From 50 to $100 \mathrm{mg}$ of liver pieces were incubated in $400 \mu$ l of hypotonic buffer (10 mM HEPES-KOH, pH 7.4, 10 mM KCl, $1.5 \mathrm{mM} \mathrm{MgCl}_{2}, 0.5 \mathrm{mM}$ DTT, protease and phosphatase inhibitor cocktails; Roche) and lysed with 10 strokes in a Dounce homogenizer. The supernatant fraction containing mainly cytoplasmic proteins was collected after centrifugation $\left(14,000 \mathrm{~g}\right.$ for 5 minutes, $4^{\circ} \mathrm{C}$ ), and the pellets were washed twice with hypotonic buffer. The pellets were resuspended in 100 to $200 \mu \mathrm{l}$ of hypertonic buffer (50 mM Tris- $\mathrm{HCl} \mathrm{pH} 7.4,150 \mathrm{mM} \mathrm{NaCl}, 0.1 \%$ NP-40, protease and phosphatase inhibitor cocktails) for 30 minutes on ice. The supernatant containing mainly nucleoplasm and membrane fractions was collected by centrifugation (2,000 $g$ for 5 minutes, $4^{\circ} \mathrm{C}$ ), and the remaining insoluble pellet containing mainly chromatin was resuspended in $100 \mu$ l of hypertonic buffer and sonicated. Before running SDS-PAGE, protein lysates were boiled for 5 minutes at $95^{\circ} \mathrm{C}$. The following proteins were used for Western blotting: anti-Hsp90, anti-SREBP-1, and anti-P62 (BD Biosciences catalog 610418, 557036, and 610497); anti-OSBPL3 (Novus Biologicals, catalog NBP-155151), anti-FASN (Abcam, catalog ab22759), anti-SCD1 (Thermo Fisher, catalog A13996), anti- $\alpha$-tubulin, anti-PARP1, and anti- $\beta$-actin (Santa Cruz Biotechnology Inc., catalog sc-5286, sc-7150, and sc-47778). Anti-SREBP-1, anti-P62, and anti-PARP1 were blotted on nuclear/ membrane fractions and all other antibodies on cytoplasmic fractions.

Gene expression and analysis. RNA was extracted from the livers of mice that were fasted for 24 hours followed by 6 hours of refeeding, LRH-1 WT ( $n=6)$ and LRH-1 K289R $(n=6)$. Extraction was performed using TRIZOL (Invitrogen) and purified with the RNeasy Cleanup Kit for Microarray Analysis (QIAGEN). For quantitative reverse-transcription PCR (RT-qPCR), cDNA was generated using the QuantiTect Reverse Transcription Kit (QIAGEN) and analyzed by qPCR using a LightCycler 480 Real-Time PCR System (Roche) and the primers listed in Supplemental Table 1. Expression data were normalized to $36 b 4$ or B2m mRNA levels. Microarray analysis was performed using the Affymetrix MouseGene 1.0 ST array and normalized using the robust multi-array average (RMA) method. All original microarray data were deposited in the NCBI's Gene Expression Omnibus (GEO GSE89877). Heat maps were generated using GENE-E (http://www.broadinstitute. org/cancer/software/GENE-E/index.html), and rows were clustered using the one minus Pearson correlation metric.

ChIP-PCR. ChIP analysis was performed as described previously, with minor modifications (9). ChIPed DNA was purified using the PCR Clean-up Extraction Kit (Macherey-Nagel), after which qPCR was performed as described previously (47). Data were normalized to

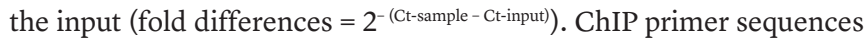
are listed in Supplemental Table 2. For LXR ChIP assays, an anti-LXR $\alpha$ antibody (Abcam, catalog ab41902) was used. For LRH-1 ChIP experiments, a custom LRH-1 antibody was generated. A synthetic peptide ([H]-QEQSNRNRQEKLSAFG-[NH2]) was used to immunize 2 rab- 
bits (AbFRONTIER). The antiserum with the highest ELISA titer was selected and used in ChIP experiments. The preimmunization serum from the same rabbit was used as a negative control.

LXR agonist treatment. Hepa 1.6 (ATCC, catalog CRL1830) or AML-12 (ATCC, catalog CRL2254) cells were treated with $1 \mu \mathrm{M}$ of GW3965 (Selleckchem), a dual LXR $\alpha$ and LXR $\beta$ agonist, for 6 hours in full medium.

In vivo siRNA transfection. siRNA sequences are listed in Supplemental Table 4. HPLC-purified siRNAs ( 3 nmol, Microsynth) were tailvein injected into each recipient mouse using in vivo-JetPEI (Polyplus) according to the manufacturer's instructions. Mice were fast-refed as described above and sacrificed 2 days after injection.

Adenoviral infection. Osbpl3 cDNA was cloned into a pENTR/ D-TOPO plasmid (Invitrogen; the Topo primer is listed in Supplemental Table 3) and then subcloned in the pAd/CMV-DEST plasmid (Invitrogen). After linearization with $\mathrm{PacI}$, the construct was transfected into HEK 293A cells to produce the adenoviruses. Mice were tail-vein injected with Ad-gfp or Ad-Osbpl3, $2.5 \times 10^{9} \mathrm{PFU}$, fast-refed as described above, and sacrificed 3 days after injection.

AAV8 miRNA injection. The Osbpl3-siRNA sequence (Supplemental Table 4) was engineered to create a pre-miRNA sequence targeting Osbpl3, which was cloned into an AAV vector construct driven by the liver-specific $h A A T$ promoter. AAV8 mirOsbpl3 viral vectors were generated and titrated as described previously $(48,49)$ and injected into jugular vein under isoflurane anesthesia $\left(5 \times 10^{11} \mathrm{vg}\right)$. Two weeks after the injection, mice were fasted for 24 hours followed by 6 hours refeeding, then sacrificed, and tissues were snap-frozen.

Lipid analyses and plasma parameters. Hepatic lipids were extracted according to the Bligh and Dyer protocol (50). Triglycerides, free fatty acids, and cholesterol contents in plasma and/or hepatic lipid fractions were quantified using enzymatic assays (Roche). To asses fatty acid synthesis rates in vivo, mice received sodium $\left[1{ }^{13} \mathrm{C}\right]$ acetate via their drinking water (2\%) 24 hours prior to sacrifice. Fatty acids derived from hepatic lipid extracts were liberated, derivatized, and subjected to gas chromatography-mass spectrometry (GC-MS) analysis in order to calculate the fractional synthesis rates from the incorporation of ${ }^{13} \mathrm{C}$-acetate as described previously (51). ASAT and ALAT concentrations in blood were determined using standard clinical chemistry methods.

$V L D L$ secretion assay and glucose-6-phosphate quantification. The VLDL secretion assay was performed as described previously (51). Thirteen-week-old male LRH-1 K289R or LRH-1 WT mice were subjected to the fasting-refeeding protocol and treated with $50 \mathrm{mg} / \mathrm{ml}$ poloxamer 407 (Sigma-Aldrich, catalog 16758) 8 hours after refeeding was initiated. Blood samples were collected once before and every hour after poloxamer treatment to determine the triglyceride content. Glucose-6-phosphate content was determined using enzymatic assays as described previously (6).
Immunohistochemistry. Liver sections were cut into 5 - $\mu$ m-thick serial cryosections for oil red $\mathrm{O}$ staining to visualize neutral lipids or paraffin cryosections to stain collagen with sirius red, CD45-positive cells using rat anti-CD45 antibody (eBioscience, catalog 30-F11), or H\&E.

Statistics. Data are expressed as mean \pm SEM. Comparison of differences between 2 groups was assessed using unpaired 2-tailed Student's $t$ tests. Multiple group comparisons were assessed by ANOVA and Bonferroni's post-hoc $t$ tests. $P<0.05$ was considered statistically significant.

Study approval. All animal procedures were approved by the Swiss authorities (Canton of Vaud, animal protocols ID 2380, 2561, and 2768) and performed in accordance with École Polytechnique Fédérale de Lausanne institutional guidelines.

\section{Author contributions}

SS and VL designed and carried out most of the experiments, analyzed data, prepared the figures, and wrote the manuscript. PX and DR helped with Western blotting, histology, adenovirus production, and/or in vivo experiments. HD carried out the ChIPqPCR experiments. XW helped with bioinformatic analyses. VJ and FB designed and generated the AAV8 constructs. MHO performed GC-MS analysis and quantification of fatty acids. TFL and MHO provided useful advice on the experimental procedures. KS supervised all aspects of the work.

\section{Acknowledgments}

We thank Vasco de Campos and Norman Moullan for help with tailvein and jugular-vein injections and Theo van Dijk, Theo Boer, Sara Oppi, Thibaud Clerc, Sabrina Bichet, and Soline Odouard fortechnical help. This study was funded by École Polytechnique Fédérale de Lausanne and the Swiss National Science Foundation (31003A_1666695 and CRSII3_160798/1). SS was supported by postdoctoral fellowships from the German Academy of Sciences Leopoldina (LPDS 2011-6) and the Novartis Consumer Health Foundation and an Ambizione grant from the Swiss National Science Foundation (PZOOP3 161521). VL was supported by a PhD grant from the Portuguese Foundation for Science and Technology (SFRH/BD/52046/2012) through the Graduate Program in Basic and Applied Biology (GABBA) PhD program. MHO holds a Rosalind Franklin Fellowship from the University of Groningen. FB is an ICREA Academia recipient, Generalitat de Catalunya, Spain. AAV vector generation and production were funded by the Ministerio de Economía y Competitividad, Plan Nacional I+D+I (SAF2014-54886-R), Spain.

Address correspondence to: Kristina Schoonjans, Institute of Bioengineering, School of Life Sciences, École Polytechnique Fédérale de Lausanne, EPFL SV SSV-GE, AI 1149 (Bâtiment AI), Station 19, CH-1015 Lausanne, Switzerland. Phone: 41.21.693.18.91; E-mail: kristina.schoonjans@epfl.ch.
1. Cohen JC, Horton JD, Hobbs HH. Human fatty liver disease: old questions and new insights. Science. 2011;332(6037):1519-1523.

2. Villanueva A, Llovet JM. Liver cancer in 2013: Mutational landscape of HCC--the end of the beginning. Nat Rev Clin Oncol. 2014;11(2):73-74.

3. Angulo P. Nonalcoholic fatty liver disease. $N$ Engl JMed. 2002;346(16):1221-1231.
4. Perry RJ, Samuel VT, Petersen KF, Shulman GI. The role of hepatic lipids in hepatic insulin resistance and type 2 diabetes. Nature. 2014;510(7503):84-91.

5. Stein S, Schoonjans K. Molecular basis for the regulation of the nuclear receptor LRH-1. Curr Opin Cell Biol. 2015;33:26-34.

6. Oosterveer MH, et al. LRH-1-dependent glucose sensing determines intermediary metabolism in liver. J Clin Invest. 2012;122(8):2817-2826.

7. Lee JM, et al. A nuclear-receptor-dependent phosphatidylcholine pathway with antidiabetic effects. Nature. 2011;474(7352):506-510.

8. Musille PM, Pathak MC, Lauer JL, Hudson WH, Griffin PR, Ortlund EA. Antidiabetic phospholipid-nuclear receptor complex reveals the mech- 
anism for phospholipid-driven gene regulation. Nat Struct Mol Biol. 2012;19(5):532-537.

9. Stein S, et al. SUMOylation-dependent LRH-1/ PROX1 interaction promotes atherosclerosis by decreasing hepatic reverse cholesterol transport. Cell Metab. 2014;20(4):603-613.

10. Keeton AB, Bortoff KD, Bennett WL, Franklin JL, Venable DY, Messina JL. Insulin-regulated expression of Egr-1 and Krox2O: dependence on ERK1/2 and interaction with p38 and PI3-kinase pathways. Endocrinology. 2003;144(12):5402-5410.

11. Keeton AB, Bortoff KD, Franklin JL, Messina JL. Blockade of rapid versus prolonged extracellularly regulated kinase $1 / 2$ activation has differential effects on insulin-induced gene expression. Endocrinology. 2005;146(6):2716-2725.

12. Chung J, et al. Intracellular transport. PI4P/phosphatidylserine countertransport at ORP5- and ORP8-mediated ER-plasma membrane contacts. Science. 2015;349(6246):428-432.

13. Moser von Filseck J, et al. Intracellular transport. Phosphatidylserine transport by ORP/Osh proteins is driven by phosphatidylinositol 4-phosphate. Science. 2015;349(6246):432-436.

14. Maeda K, et al. Interactome map uncovers phosphatidylserine transport by oxysterol-binding proteins. Nature. 2013;501(7466):257-261.

15. Yan D, et al. Oxysterol binding protein induces upregulation of SREBP-1c and enhances hepatic lipogenesis. Arterioscler Thromb Vasc Biol. 2007;27(5):1108-1114.

16. Holmstrom SR, et al. LRH-1 and PTF1-L coregulate an exocrine pancreas-specific transcriptional network for digestive function. Genes Dev. 2011;25(16):1674-1679.

17. Chong HK, Biesinger J, Seo YK, Xie X, Osborne TF. Genome-wide analysis of hepatic LRH-1 reveals a promoter binding preference and suggests a role in regulating genes of lipid metabolism in concert with FXR. BMC Genomics. 2012;13:51.

18. Venteclef N, Smith JC, Goodwin B, Delerive P. Liver receptor homolog 1 is a negative regulator of the hepatic acute-phase response. Mol Cell Biol. 2006;26(18):6799-6807.

19. Venteclef N, et al. GPS2-dependent corepressor/SUMO pathways govern anti-inflammatory actions of LRH- 1 and LXRbeta in the hepatic acute phase response. Genes Dev. 2010;24(4):381-395.

20. Cha JY, Repa JJ. The liver X receptor (LXR) and hepatic lipogenesis. The carbohydrate-response element-binding protein is a target gene of LXR. J Biol Chem. 2007;282(1):743-751.

21. Joseph SB, et al. Direct and indirect mechanisms for regulation of fatty acid synthase gene expression by liver X receptors. J Biol Chem. 2002;277(13):11019-11025.
22. Matsukuma KE, Wang L, Bennett MK, Osborne TF. A key role for orphan nuclear receptor liver receptor homologue- 1 in activation of fatty acid synthase promoter by liver X receptor. J Biol Chem. 2007;282(28):20164-20171.

23. Huang J, et al. Molecular characterization of the role of orphan receptor small heterodimer partner in development of fatty liver. Hepatology. 2007;46(1):147-157.

24. Duval C, et al. Adipose tissue dysfunction signals progression of hepatic steatosis towards nonalcoholic steatohepatitis in C57BL/6 mice. Diabetes. 2010;59(12):3181-3191.

25. Moylan CA, et al. Hepatic gene expression profiles differentiate presymptomatic patients with mild versus severe nonalcoholic fatty liver disease. Hepatology. 2014;59(2):471-482.

26. Ahrens M, et al. DNA methylation analysis in nonalcoholic fatty liver disease suggests distinct disease-specific and remodeling signatures after bariatric surgery. Cell Metab. 2013;18(2):296-302.

27. Yin C, Evason KJ, Asahina K, Stainier DY. Hepatic stellate cells in liver development, regeneration, and cancer. J Clin Invest. 2013;123(5):1902-1910.

28. Venteclef N, Delerive P. Interleukin-1 receptor antagonist induction as an additional mechanism for liver receptor homolog-1 to negatively regulate the hepatic acute phase response. J Biol Chem. 2007;282(7):4393-4399.

29. Horton JD, Goldstein JL, Brown MS. SREBPs: activators of the complete program of cholestero and fatty acid synthesis in the liver. JClin Invest. 2002;109(9):1125-1131.

30. Krycer JR, Sharpe LJ, Luu W, Brown AJ. The AktSREBP nexus: cell signaling meets lipid metabolism. Trends Endocrinol Metab. 2010;21(5):268-276.

31. Yecies JL, et al. Akt stimulates hepatic SREBP1c and lipogenesis through parallel mTORC1dependent and independent pathways. Cell Metab. 2011;14(1):21-32.

32. Haas JT, et al. Hepatic insulin signaling is required for obesity-dependent expression of SREBP-1c mRNA but not for feeding-dependent expression. Cell Metab. 2012;15(6):873-884.

33. Leavens KF, Easton RM, Shulman GI, Previs SF, Birnbaum MJ. Akt2 is required for hepatic lipid accumulation in models of insulin resistance. Cell Metab. 2009;10(5):405-418.

34. Ricoult SJ, Yecies JL, Ben-Sahra I, Manning BD. Oncogenic PI3K and K-Ras stimulate de novo lipid synthesis through mTORC1 and SREBP. Oncogene. 2016;35(10):1250-1260.

35. Walker AK, et al. A conserved SREBP-1/phosphatidylcholine feedback circuit regulates lipogenesis in metazoans. Cell. 2011;147(4):840-852.

36. Kandutsch AA, Shown EP. Assay of oxysterolbinding protein in a mouse fibroblast, cellfree system. Dissociation constant and other properties of the system. J Biol Chem 1981;256(24):13068-13073.

37. Dawson PA, Ridgway ND, Slaughter CA, Brown MS, Goldstein JL. cDNA cloning and expression of oxysterol-binding protein, an oligomer with a potential leucine zipper. J Biol Chem. 1989;264(28):16798-16803.

38. Dawson PA, Van der Westhuyzen DR, Goldstein JL, Brown MS. Purification of oxysterol binding protein from hamster liver cytosol. J Biol Chem. 1989;264(15):9046-9052.

39. Ridgway ND. Oxysterol-binding proteins. Subcell Biochem. 2010;51:159-182.

40. Olkkonen VM, Li S. Oxysterol-binding proteins: sterol and phosphoinositide sensors coordinating transport, signaling and metabolism. Prog Lipid Res. 2013;52(4):529-538.

41. Weber-Boyvat M, et al. OSBP-related protein 3 (ORP3) coupling with VAMP-associated protein A regulates R-Ras activity. Exp Cell Res. 2015;331(2):278-291.

42. Lehto M, Hynynen R, Karjalainen K, Kuismanen E, Hyvärinen K, Olkkonen VM. Targeting of OSBP-related protein 3 (ORP3) to endoplasmic reticulum and plasma membrane is controlled by multiple determinants. Exp Cell Res. 2005;310(2):445-462.

43. Lehto M, et al. The R-Ras interaction partner ORP3 regulates cell adhesion. JCell Sci. 2008;121(Pt 5):695-705.

44. Cave MC, et al. Nuclear receptors and nonalcoholic fatty liver disease. Biochim Biophys Acta. 2016;1859(9):1083-1099.

45. Neuschwander-Tetri BA, et al. Farnesoid X nuclear receptor ligand obeticholic acid for non-cirrhotic, non-alcoholic steatohepatitis (FLINT): a multicentre, randomised, placebo-controlled trial. Lancet. 2015;385(9972):956-965.

46. Sanyal AJ, et al. Pioglitazone, vitamin E, or placebo for nonalcoholic steatohepatitis. N Engl J Med. 2010;362(18):1675-1685.

47. Mataki C, et al. Compromised intestinal lipid absorption in mice with a liver-specific deficiency of liver receptor homolog 1. Mol Cell Biol. 2007;27(23):8330-8339.

48. Ayuso E, et al. High AAV vector purity results in serotype- and tissue-independent enhancement of transduction efficiency. Gene Ther. 2010;17(4):503-510.

49. Lock M, et al. Characterization of a recombinant adeno-associated virus type 2 Reference Standard Material. Hum Gene Ther. 2010;21(10):1273-1285.

50. Bligh EG, Dyer WJ. A rapid method of total lipid extraction and purification. Can J Biochem Physiol. 1959;37(8):911-917.

51. Oosterveer $\mathrm{MH}$, et al. High fat feeding induces hepatic fatty acid elongation in mice. PLoS One. 2009;4(6):e6066. 